\title{
Hemoglobin Switching in Humans Is Accompanied by Changes in the Ratio of the Transcription Factors, GATA-1 and SPI
}

\author{
E. Rena Bacon, ${ }^{* 1}$ Nava Dalyot,* Dvora Filon,* \\ Letizia Schreiber, ${ }^{\dagger}$ Eliezer A. Rachmilewitz, ${ }^{*}$ \\ and Ariella Oppenheim* \\ Departments of ${ }^{*}$ Hematology and ${ }^{\dagger}$ Pathology, Hebrew University- \\ Hadassah Medical School and Hadassah University Hospital, \\ Jerusalem, Israel
}

\begin{abstract}
Background: Understanding the mechanism of developmental regulation of hemoglobin switching has scientific as well as clinical relevance because of the influence of fetal hemoglobin $(\mathrm{HbF})$ production in adulthood on the clinical manifestation of thalassemia and sickle cell anemia. We have previously found that the normal developmental patterns of globin gene expression are recapitulated in an experimental system of primary cultures that support differentiation of erythroid progenitors. We further found that high activities of the transcriptional activators, GATA-1 and SP1, are associated with normal adult erythroid differentiation.

Materials and Methods: In the present work, we have studied the activities of GATA-1 and SPI during differentiation of cultured erythroid progenitors derived from cord blood and from fetal livers, as well as from $\beta^{\mathrm{O}}$. thalassemia patients.
\end{abstract}

Results: The results showed high GATA-1 binding ac- tivity and very low SPl activity in the fetal liver cultures. This pattern was in contrast to cultures derived from normal adult peripheral blood, in which both GATA-1 and SPl activities were high. Cord blood cultures showed an additive combination of "adult" and "fetal" patterns. The progenitors derived from a $\beta^{0}$-thalassemia patient with high $\mathrm{HbF}$ production showed "fetal" pattern. On the other hand, in cultures of $2 \beta^{0}$-thalassemia patients without high HbF, "adult" pattern was observed.

Conclusions: In the present work, we show that human fetal and adult erythroid progenitors are distinct in their transcription factors, and that the commitment to fetal or adult program occurs at a very early differentiation stage. Our studies also demonstrate that under anemic stress, recruitment of fetal progenitors may occur in adulthood.

\section{INTRODUCTION}

Elucidation of the mechanism underlying developmental regulation of hemoglobin switching has important medical implications. For example, elevated fetal hemoglobin ( $\mathrm{HbF})(\alpha 2 \gamma 2)$ production in adult patients with sickle cell anemia or $\beta$-thalassemia ameliorates the disease symp-

Address correspondence and reprint requests to: Ariella Oppenheim, Department of Hematology, Hebrew University-Hadassah Medical School, Jerusalem, Israel 91120.

${ }^{1}$ On Sabbatical leave from Ramapo College of New Jersey, Mahwah, NJ 07430-1680. toms, as the $\gamma$-globin chains may compensate for the reduction in $\beta$-globin chains. In some individuals this occurs due to $\gamma$-globin promoter mutations or to deletions in the $\beta$-globin gene cluster. In others, the mechanism is not understood.

In spite of intensive research, this mechanism has not been clarified, partly because of the unavailability of an adequate animal model, since the switch mechanism varies among different mammals. It has been suggested that normal adult erythroid cells go through a "fetal" stage during differentiation before maturing into cells producing adult hemoglobin $(\alpha 2 \beta 2)$ (1). The 
clonal model, on the other hand, invokes distinct progenitors and separate pathways (2).

Our previous studies demonstrated that adult and neonatal patterns of globin gene expression are recapitulated in liquid cultures that support proliferation and differentiation of erythroid progenitors (3). The cultures are grown in two phases: first the early progenitors, burst forming units, erythroid, (BFUe), proliferate and differentiate into colony forming units, erythroid (CFUe). Further differentiation and maturation, including globin gene expression and hemoglobin biosynthesis, is erythropoietin (EPO) dependent (4). In our experiments (3), cultured adult progenitors, from peripheral blood, gave rise primarily to the $\beta$ - and $\alpha$-globin expressing cells, while progenitors of neonate cord blood (CBL) differentiated into cells that also synthesized high levels of $\gamma$-globin mRNA. Those experiments suggested that the fetal progenitors are distinct from adult progenitors and that CBL contains a mixed population of both. The peak of $\gamma$-globin gene expression usually preceded that of $\beta$, implying that fetal progenitors tend to differentiate faster than the adult ones.

We further found that the appearance of globin mRNA in adult cultures was preceded by a burst in the level of GATA-1, which was triggered by the addition of EPO (5). GATA-1 is a major transcriptional activator of erythroid differentiation $(6,7)$, regulating a number of erythroid specific genes, including globin (8). We observed a parallel rise in the level of the ubiquitous factor, SP1, indicating that not only GATA-1, but also SP1 participates in adult erythroid maturation. Additional evidence for the role of SPl in human erythroid differentiation comes from other studies. Analysis of binding of transcriptional factors at the $\beta$-globin locus control region (LCR) suggested that the combination of SPI and GATA-1 plays a role in the high levels of globin gene expression (9). It was also found that GATA- 1 and SPI interact at the $\gamma$-globin promoter. Depending on their concentrations, this interaction led to synergistic transcriptional activation or to interference (10). Furthermore, it has been reported that the levels of GATA-1 and SP1 play an important role in the mechanism of globin switching in the chicken (11). Primary cells of the primitive erythroid lineage were found to contain 10 -fold more of these two factors than did cells of the definitive erythroid lineage.

In the present work, we analyzed the activities of GATA-1 and SP1 in primary cultures de- rived from $\mathrm{CBL}$, mid-gestation fetal livers, and adult $\beta$-thalassemia patients with high and low $\mathrm{HbF}$ production. Our results indicate that fetal and adult erythroid differentiation occurs along separate pathways.

\section{MATERIALS AND METHODS}

\section{Erythroid Cultures}

CBL samples were obtained from the Hadassah maternity ward. Liver tissues were derived from fetuses aborted at 20 weeks gestational age (because of a nonglobin genetic defect), obtained from the Department of Pathology, Hebrew University (Jerusalem, Israel). Peripheral blood samples of $\beta$-thalassemia patients were withdrawn prior to transfusions. All samples were obtained according to the Helsinki Committee guidelines.

The liver tissues were finely minced and dispersed by pipetting. The cultures were grown in two phases as previously described $(3,4)$. The dispersed liver cells, or buffy coats of the blood samples, were cultured in the presence of conditioned medium from human bladder carcinoma cells for 7 days (from Day -7 to Day 0 ). The cells were then washed and recultured in medium supplemented with $1 \mathrm{U} / \mathrm{ml}$ recombinant EPO. Microscopic slides were prepared by cytospin, following lysis of the enucleated red blood cells with $\mathrm{H}_{2} \mathrm{O}$, and stained with May-Grunwald Giemsa. Hemoglobinized cells were identified by the benzidine peroxidase reaction (12).

\section{RNA Analyses}

RNA was purified from cytoplasmic miniextracts, prepared in parallel to nuclear extracts $(13,14)$ from a total of $2 \times 10^{6}$ cells. Reverse transcription (RT) was performed with oligo(dT).

The primers for PCR were the following: for $\gamma$-globin, the $5^{\prime}$ primer was ACAAGGCTACTAT CACAAGCCT (exon 1), and the $3^{\prime}$ primer was CTTTATGGCATCTCCCAAGGAA (exon 2); for $\beta$-globin, the $5^{\prime}$ primer was CACCTTTGCCA CACTGAGTGA (exon 2), and the $3^{\prime}$ primer CCA GATGCTCAAGGCCCTTC (from the untranslated region $3^{\prime}$ to exon 3 ). The amplification cycle was $92^{\circ} \mathrm{C}$ for $1 \mathrm{~min}, 60^{\circ} \mathrm{C}$ for $1 \mathrm{~min}$, and $72^{\circ} \mathrm{C}$ for 1 min, repeated for 30 cycles. The reaction products were visualized by ethidium bromide staining following electrophoresis on $8 \%$ native acrylamide gels.

The PCR primers for the GATA-1 mRNA 
analysis were GCCCATCTCTACCAAGGCCT and AGAAGGACACCACCCCTCCT, derived from the 3' end of the human GATA-1 mRNA, producing a 204 bp amplification product (5). The PCR primers for GATA-2 analysis were CCCTAAG CAGCGCAGCAAGAC and GATGAGTGGTCGG TTCTGGCC (15). The amplification cycle for both GATA- 1 and GATA- 2 was $92^{\circ} \mathrm{C}$ for $1 \mathrm{~min}, 62^{\circ} \mathrm{C}$ for $1 \mathrm{~min}$, and $72^{\circ} \mathrm{C}$ for $1 \mathrm{~min}$, repeated for 30 cycles.

\section{Preparation of Nuclear Extracts and Gel Retardation Assays}

Nuclear miniextracts (13) were prepared from a total of $2 \times 10^{6}$ cells for each time-point. Binding activities of GATA-1 and SP1 were assayed by the gel retardation assay as previously described (5). Oligonucleotides for the gel retardation assay were the following: for GATA-1, CGGGCAACT GATAAGGATTCCCA, derived from the promoter region of the mouse $\alpha$-1 globin gene (16); and for SPI, CTTGGGGGCCCCTCCCCCACACTATCTCAAT, derived from the human $\gamma$-globin gene (this oligonucleotide contains a $\mathrm{T}$ to $\mathrm{C}$ transition at position -198 , creating a strong SP1 binding site) (17). The specific $5^{\prime}$ end-labeled oligonucleotides were annealed with an excess of the complementary oligonucleotide and repurified by polyacrylamide gel electrophoresis. Binding reactions were performed on equal amounts of nuclear proteins obtained from $1.2 \times 10^{5}$ cells. Competition experiments were performed by adding 100 -fold molar excess of unlabeled competitor oligonucleotide. The DNA-protein complexes were then separated on $5 \%$ native polyacrylamide gels.

Intensities of the shifted bands were determined by phospho-imaging of the gels using the Bio-Imaging Analyzer, BAS1000, FUJIX.

\section{RESULTS}

\section{GATA-1 and SP1 Activities in Erythroid Progenitors Derived from CBL}

To gain an insight into the mechanism of hemoglobin switching, we examined the factors GATA-l and SPI in differentiating progenitors derived from CBL. Samples of CBL were grown in the two-phase liquid culture system (4). In the first phase, mononuclear cells were cultured with conditioned medium containing hemapoietic growth factors not including EPO. EPO was added at the beginning of the second phase. Samples were taken at different time points, and nuclear extracts were prepared. We found that, similar to the adult cultures, high levels of GATA-1 binding activity were present on Days 7-12 (Fig. la and b). However, while the level of SP1 in the adult cultures rose in parallel to that of GATA-1 (5) (see also Fig. 3d), in the CBL culture SP1 was very low on Days 7 and 9, and increased substantially, to adult level, only on Day 12 . These observations are consistent with the notion that CBL contains a mixture of adult and fetal progenitors. We concluded that the high GATA-1 and SP1 (seen on Day 12) characterize the adult progenitors present in the culture, which appear to differentiate more slowly than the fetal progenitors. We attributed the high GATA-1/low SP1 activities seen on Days 7-9 to the differentiating fetal cells. Therefore, the results implied that the fetal erythroid differentiation program is associated with high GATA-1 activity and low SP1.

\section{Differentiation of Fetal Liver Progenitors in Liquid Culture}

To obtain more direct evidence we proceeded to investigate differentiating erythroid cultures derived from midgestation human fetal livers, as a source of pure fetal progenitors. Livers were obtained from aborted fetuses (Week 19-20 gestational age) and single-cell suspensions were prepared. Those included a large proportion of nucleated, hemoglobinized erythroid cells (orthochromatic normoblasts). The cells were plated with conditioned medium (Day -7). Three days later (Day -4 ) a small number of nonhemoglobinized erythroid cells (pronormoblasts and basophilic normoblasts) appeared. Those were presumably cells that were beyond the CFUe stage, and already EPO-induced, when placed in culture. During the first phase, the number of the nucleated erythroid cells decreased dramatically, due to enuclation of the orthochromatic normoblasts and cell death. The early erythroblasts, which were visible on Day -4 , did not give rise to hemoglobinized cells, since they probably died in the absence of EPO. After EPO addition (Day 0) significant erythroid proliferation and differentiation ensued. The differentiation of a representative culture after EPO addition is shown in Fig. 2a.

Globin mRNA was analyzed by the RT-PCR procedure. As seen in Fig. $2 \mathrm{~b}$, cells from the first phase contained both $\beta$ - and $\gamma$-mRNA (Days -4 

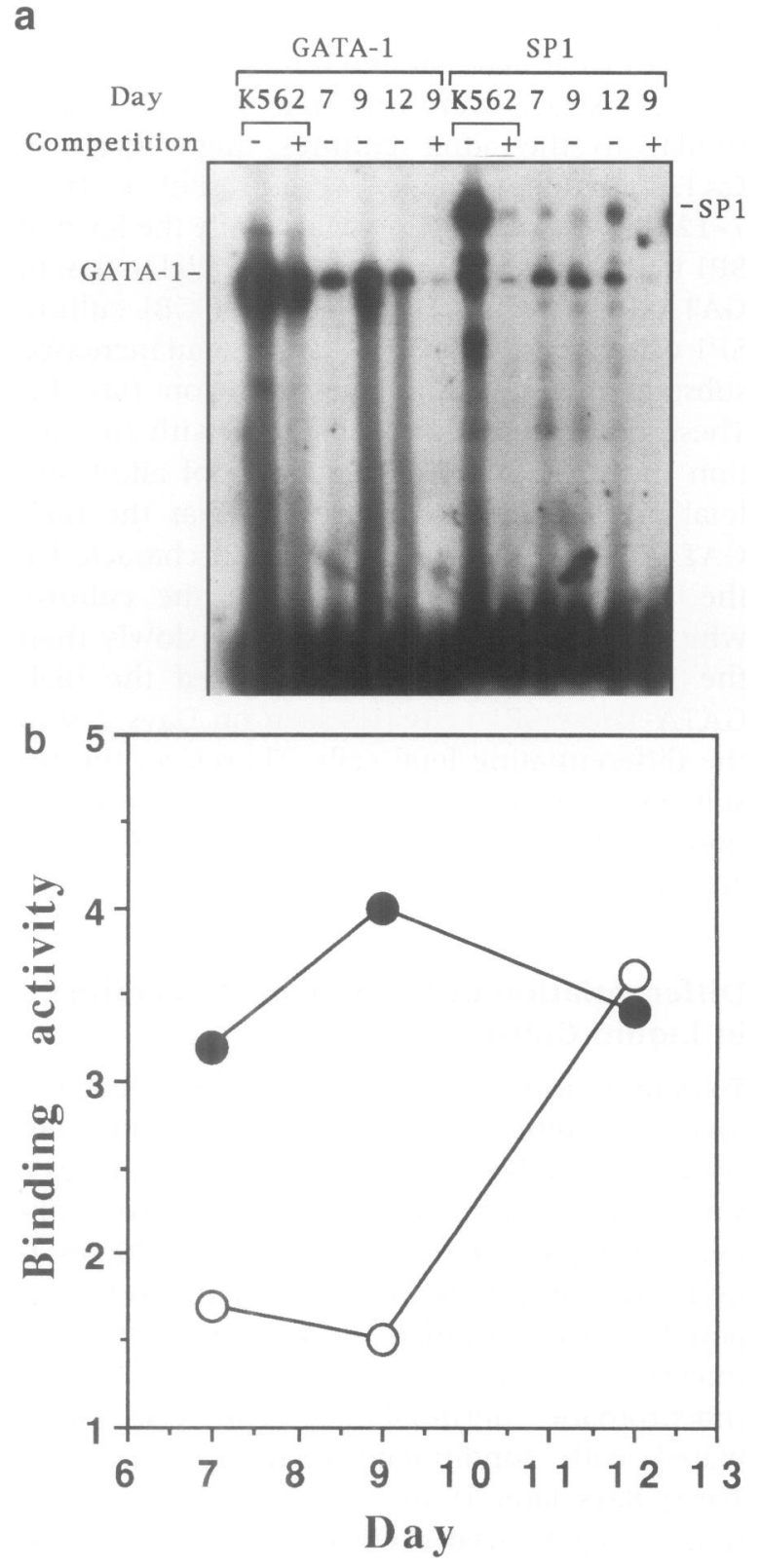

FIG. 1. Analysis of transcription factors GATA-1 and SPI in CBL culture, second phase

(a) Autoradiogram of gel retardation assay. The numbers on top represent days in the second phase, after EPO addition. K562, an erythroleukemia cell line serving as a positive control; competition, binding in the presence of 100 -fold molar excess of unlabeled competitor oligonucleotide. The lower band in the SPl retardation assay, which comigrates with the GATA-1-shifted band, is probably due to nonspecific binding (17). (b) Quantitative analysis of the autoradiograms. GATA-l binding activity; $\bigcirc$, SPl binding activity. and 0 ), suggesting that they included both fetaltype and adult-type cells. The adult-type cells in the fetal liver were presumably derived from the circulating fetal blood. At this gestational age, approximately $10 \%$ of the hemoglobin in the fetal blood is adult hemoglobin (normal $\beta / \gamma$ chain synthesis ratio is $\sim 10 \%$ [18]). In the second phase, the cells contained only $\gamma$-globin mRNA (Fig. 2c, Days 5 and 8), indicating that those cells were derived from pure fetal progenitors. The $343 \mathrm{bp}$ band corresponds to the unspliced $\gamma$-RNA (or DNA).

\section{Analyses of GATA-1 and SP1 Activities in the Fetal Liver Cultures}

Nuclear proteins were extracted and assayed for the levels of GATA-1 and SPl by the gel retardation assay using end-labeled double-stranded oligonucleotides containing either binding site. The results obtained from the fetal liver culture shown in Fig. 2 are presented in Fig. 3a and b, and summarized graphically in Fig. 3c. Two additional fetal liver cultures gave similar results (not shown). EPO was added on Day 0. This was followed by an increase in the level of GATA-1, with a peak on Day 6, while the level of SP1 remained low throughout the culture period, in contrast to adult cultures, in which SP1 activity was significantly higher and its kinetics paralleled that of GATA-1 (compare Fig. $3 \mathrm{c}$ and d). For comparison, binding activity of GATA-1 and SP1 of a normal adult-derived culture grown, harvested and analyzed in parallel to the fetal liver culture is shown in Lanes 14-15 (Days 5 and 7) in Fig. 3a and b. In the fetal liver cells harvested on Day -7 , when the culture first started, the levels of both transcription factors were high, consistent with the presence of adulttype $\beta$-globin expressing cells. The levels of both transcription factors decreased as those (presumably BM-derived) cells matured.

Before replating the cells in the second phase (Day 0), the culture was divided, and part was grown without EPO. Neither GATA-1 nor SP1 activities were detected in the absence of EPO throughout the culture period (Lanes 16-19, Fig. 3a and b). Those nuclear extracts were positive for the ubiquitous transcription factor OTF-1 (data not shown), which served as an internal control for nuclear protein extraction. These results corroborate that GATA-1 binding activity is EPO dependent.

The experiments demonstrate that high SP1 activity is not involved in late fetal erythroid 

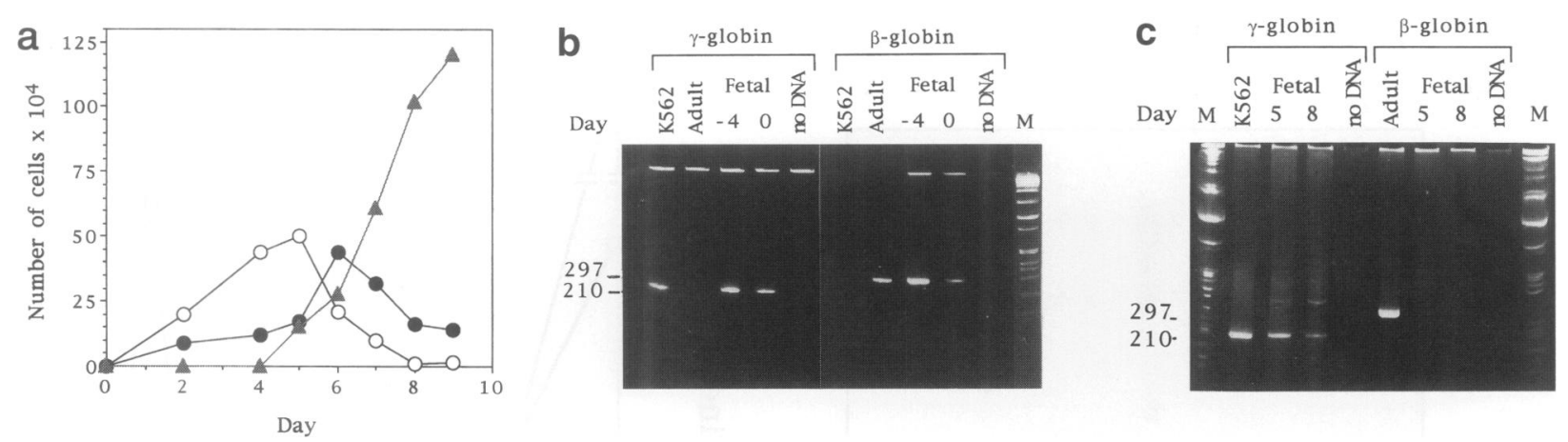

FIG. 2. Differentiation of cultured erythroid progenitors derived from fetal liver

(a) Morphological studies, second phase. The graphs represent the number of cells per $2 \times 10^{6}$ nucleated cells harvested at each time point after the addition of EPO on Day $0 . \bigcirc$, nonhemoglobinized erythroid cells (pronormoblasts and basophilic normoblasts); 0 , Partially hemoglobinized erythroid cells (polychromatophilic normoblasts); $\Delta$, Fully hemoglobinized cells (orthochromatic normoblasts). (b and c) RNA analyses of first and second phase of the fetal liver culture, respectively. Total cytoplasmic RNA was harvested and aliquots of the same RNA samples were analyzed by RT-PCR for either $\gamma$-globin mRNA (210 bp amplification product) or for $\beta$-globin mRNA (297 $\mathrm{bp})$. The numbers represent days in culture. K562 RNA served as a positive control for $\gamma$-mRNA; adult culturederived RNA, purified from a sample harvested on Day 5, served as a positive control for $\beta$-mRNA. M, DNA size marker.

differentiation. We, therefore, concluded that fetal progenitors are distinct from adult progenitors not only in their globin mRNA species but also at the level of transcription factors.

The transcription factor GATA-2 has the same binding specificity as GATA-1. It has been demonstrated, by gene targeting experiments in mouse embryonic stem cells, that GATA-2 plays a critical role in early hematopoiesis, particularly of the adult type (19). We tested the fetal culture for presence of GATA-2 RNA by RT-PCR (15). Some GATA-2 mRNA was observed during the first phase of the culture, supporting the suggested role of GATA-2. After EPO addition, only GATA- 1 and no GATA-2 mRNA was detected (data not shown).

\section{Recruitment of Fetal Progenitors in Adulthood}

Some forms of high $\mathrm{HbF}$ production in adults are associated with anemic stress, and are thus expressed only in patients with $\beta$-thalassemia or sickle cell anemia but not in their parents or carrier siblings. A possible mechanism is the recruitment of fetal cells, induced by the anemic stress. The ability to distinguish between fetal and adult erythroid cells at the level of transcription factor allows the testing of this hypothesis.

We analyzed GATA-1 and SP1 in erythroid cultures derived from peripheral blood of three $\beta^{0}$-thalassemia patients, carrying $\beta^{0}$ mutations.
These usually have severe clinical presentation because of the complete absence of $\beta$-globin chains. Patient A (Fig. 4a), homozygous for the $\beta$-globin mutation IVS2-1, has a mild form of thalassemia (intermedia) owing to very high $\mathrm{HbF}$ production $(\gamma / \alpha$ chain synthesis ratio 0.42 at the age of 27 years). His high $\mathrm{HbF}$ production in adulthood is not due to $\gamma$-globin promoter mutations but to an unidentified factor that functions in the presence of anemic stress (20). As seen in Fig. 4a, his cultured erythroid cells present a "fetal" pattern (high GATA-1/low SP1 levels), supporting the model of persistent recruitment of fetal progenitors in adulthood. Additional evidence comes from other parameters associated with fetal erythropoiesis: high titer of $\mathrm{i}$ antigen, an elevated level of $\mathrm{G} \gamma(42 \%)$ and extensive $(40 \%) \gamma$-globin DNA hypomethylation (in his erythroid cells) (21). On the other hand, his niece (Patient $\mathrm{B}$, Fig. $4 b$ ), who is also homozygous for the same $\boldsymbol{\beta}$-globin mutation, has a severe clinical form of thalassemia (major) without high $\mathrm{HbF}(\gamma / \alpha$ chain synthesis ratio 0.24 at the age of 2 years). Because of the low number of cells, only two time points were analyzed. Her culture differentiated rapidly, and the samples represent the later stages of erythroid differentiation. Nevertheless, it can be seen that she presents "adult" pattern of transcription factors (significant SP1 activity, which parallels GATA-1). Likewise, an unrelated patient (Patient C, Fig. 4c) with a $\beta$-globin genotype IVS2-1/FS44 and low 
a
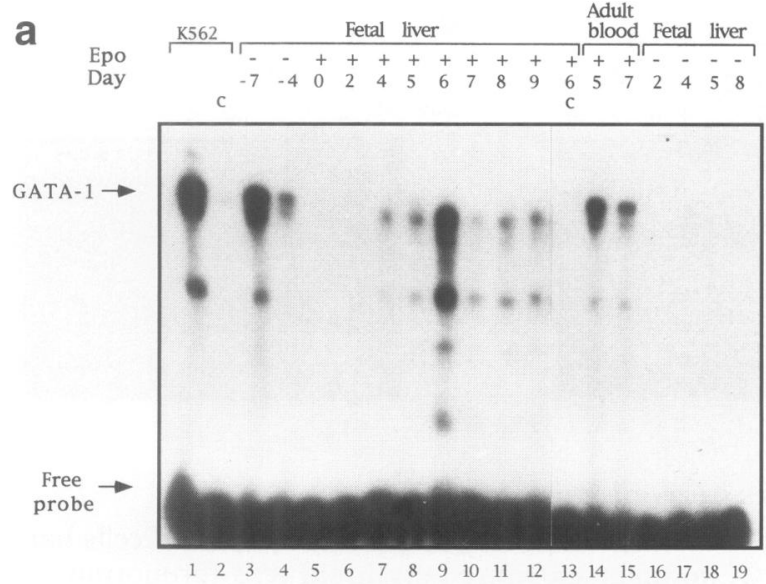

b

b
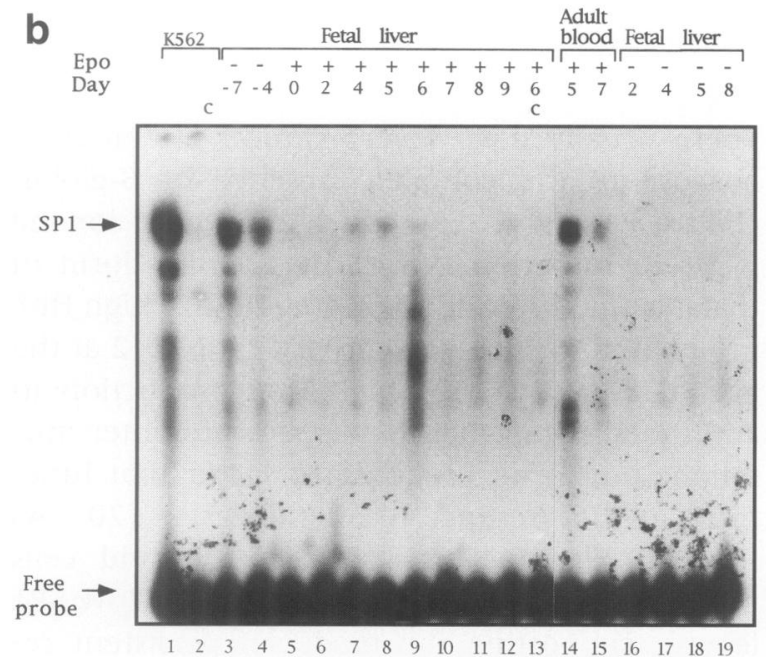

C
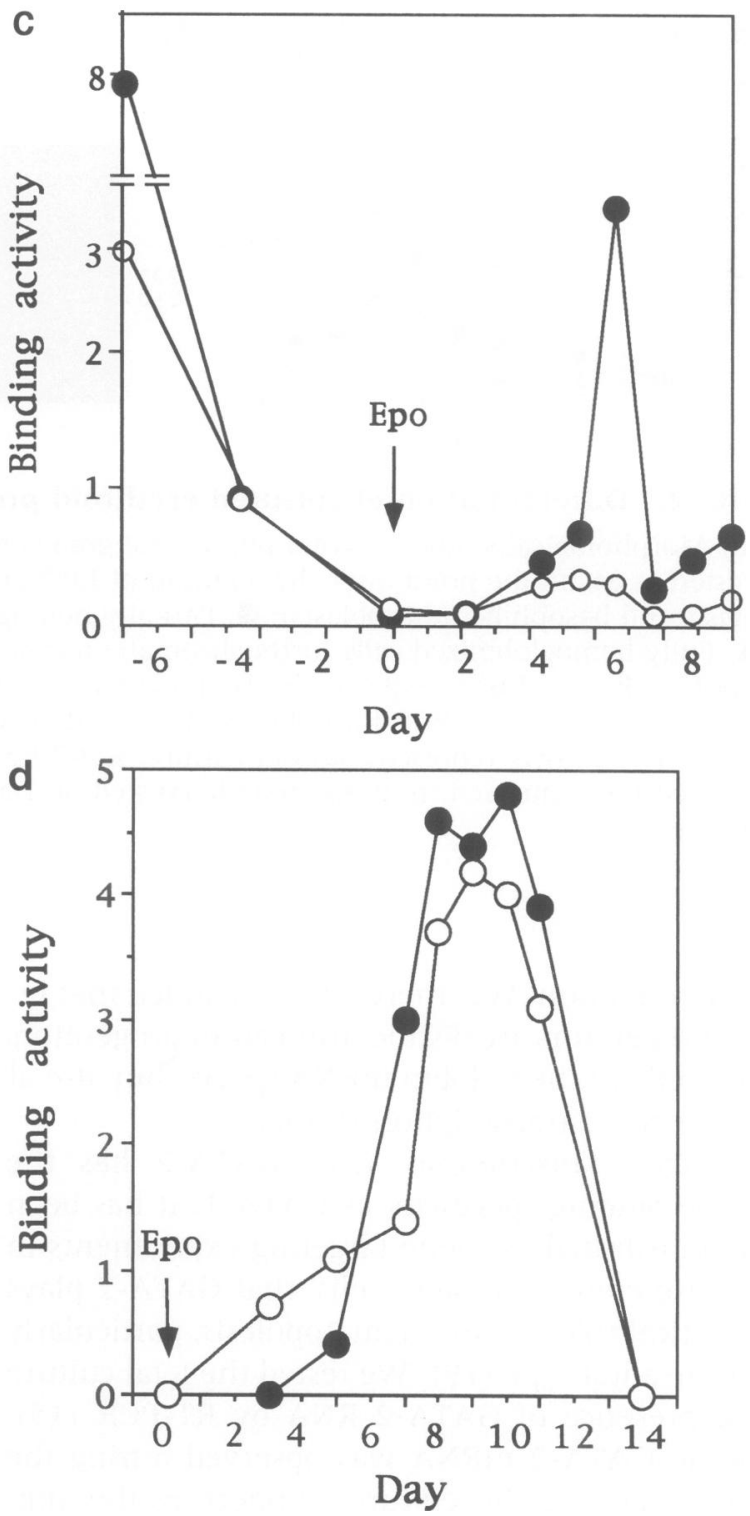

FIG. 3. Transcription factors GATA-l and SPI in differentiating fetal erythroid cells

The free probe and the shifted bands are indicated by the arrows. The day of harvest and EPO addition are shown above. The lanes are numbered below. (a) GATA-1 binding activity. (b) SPI binding activity. Lanes 1 and 2, nuclear extracts of $\mathrm{K} 562$ cells as a positive control; Lanes 2 and 13 (designated c), cold competition-binding in the presence of 100-fold molar excess of unlabeled oligonucleotide; Lanes 3-13, fetal liver cultures, first and second phase; Lanes 14 and 15, binding activity of nuclear extracts of a normal adult-derived culture grown, harvested and analyzed in parallel to the fetal liver culture; Lanes 16-19, nuclear extracts of a parallel culture of the same liver, grown in the second phase in the absence of EPO. (c) Quantification of the autoradiograms (shown in a and b). (d) GATA- 1 and SPI binding activities of a normal adult-derived culture. $\bullet$, GATA- 1 binding activity; O, SPI binding activity.

$\mathrm{HbF}(\gamma / \alpha$ chain synthesis ratio of 0.24$)$ also shows "adult" pattern. It appears that not all $\beta$-thalassemia patients are capable of significant recruitment of fetal progenitors. Further studies are needed to clarify the mechanism underlying this phenomenon.

\section{DISCUSSION}

Our studies demonstrate that erythroid progenitors derived from fetal liver have a genetic program that is distinct from that of adult-derived cells. In response to EPO, the fetal erythroblasts 

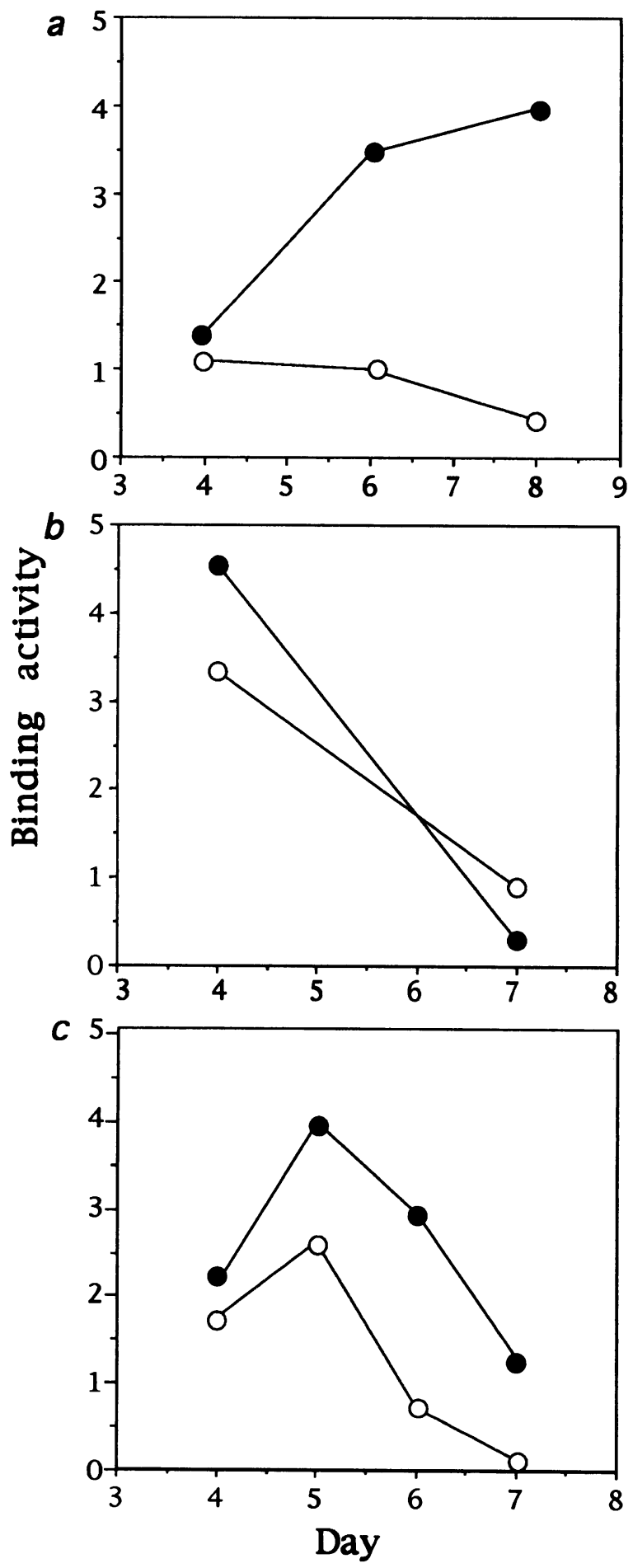

FIG. 4. GATA-1 and SPl activities in erythroid cultures derived from $\beta^{\mathbf{0}}$-thalassemia patients

(a) Patient A has thalassemia intermedia due to elevated HbF production. (b) Patient B is the niece of Patient A. She is transfusion dependent $\beta$-thalassemia major. (c) An unrelated $\beta$-thalassemia major patient. - GATA-1 binding activity; $\bigcirc$, SP1 binding activity. express a high level of GATA-1 but only a low level of SP1, in contrast to adult-derived cells, in which both transcription factors rise in parallel. Moreover, we observed good correlation between the patterns of these transcription factors and the RNA species present. High GATA-1 and high SPl correlated with $\beta$-globin mRNA expression, as was seen both in the first phase of the fetal liver cultures and in the later stage of the CBL culture (Fig. 1, Day 12). On the other hand, high GATA-1 and low SP1 were associated with only $\gamma$-globin mRNA expression, as observed in the second phase of the fetal liver cultures.

Our experiments further indicate that the fetal and adult progenitors retain their identities when present together in the same environment in the CBL cultures. Thus, commitment to the adult versus the fetal program is determined at a very early stage of differentiation, probably preBFUe, before the progenitors are placed in culture. It is possible that the fate of the progenitor is determined by effector cells present in the respective erythropoietic organ (liver versus bone marrow).

Hereditary persistence of fetal hemoglobin (HPFH) in adulthood may be induced by several mechanisms. These include mutations in cis to the $\gamma$-globin genes (promoter point mutations and large deletions in the $\beta$-globin gene cluster) as well as unlinked genetic factors (22 and references cited therein). In addition, $\mathrm{HbF}$ production may be induced by anemic stress. The pattern of transcription factors in the differentiating erythroid cells of Patient $\mathrm{A}$, with the high $\mathrm{HbF}$, provides strong evidence for the activation of fetal progenitors. However, the analyses of erythroid cultures of the other patients indicate that this is not a general phenomenon. We have previously found that the genetic factor that allows high HbF production in Patient $\mathrm{A}$ is unlinked to the $\beta$-globin gene cluster (20).

The level of $\mathrm{HbF}$ production in patients with sickle cell anemia and $\beta$-thalassemia is of high clinical significance, since $\gamma$-globin peptides may alleviate the disease symptoms. Several agents, such as hydroxyurea and butyrate derivatives, have been shown to increase $\gamma$-globin gene expression in adult patients. $\mathrm{HbF}$ and F-reticulocyte levels in sickle cell anemia patients were found to increase significantly $48-72 \mathrm{hr}$ after the administration of hydroxyurea began (23). Similarly, $\gamma$-globin synthesis followed the administration of butyrate closely (24). These findings suggest that the function of these drugs was exerted on cells that were already well on the maturation path- 
way, after the point of commitment to the adult program. Furthermore, experiments in erythroid cultures (grown similarly to those studied here) showed that both hydroxyurea and butyrate derivatives significantly elevated $\gamma$-globin expression when added to the cultures 4 days after EPO $(25,26)$, beyond the CFUe stage. We suggest that the activation of $\mathrm{HbF}$ by these agents is not via recruitment of fetal progenitors, but by a fundamentally different mechanism. We hypothesize that the adult progenitors are capable of increased $\mathrm{HbF}$ production when appropriately triggered. In the adult progenitor, the complete $\beta$-globin gene domain has an "open" chromatin configuration due to the activity of the LCR $(27,28)$. We postulate that this "open" chromatin state, in the absence of specific repressors (such as the $\varepsilon$-globin gene silencer [29]), allows $\gamma$-globin gene expression to be turned on in response to nonspecific, general gene activators. Further studies are needed to clarify this issue, which has important therapeutic implications.

\section{ACKNOWLEDGMENTS}

We are grateful to Prof. Eitan Fibach and Dr. Deborah Rund for many helpful and stimulating discussions, to Dr. Arik Oppenheim for a critical review of the manuscript, and to Ms. Merav Ginsberg for participating in preliminary studies. Dr. Antonella Ronchi provided oligonucleotides for GATA-1 and SPl analyses. We wish to thank Dr. Ilana Ariel for assistance in obtaining fetal liver samples, the staff at the Hadassah Hematology Day Care Unit for patients' PBL samples, and the staff at the Maternity Ward for CBL samples. This work was supported by Grant 91-00055 from the United States-Israel Binational Science Foundation (BSF), Jerusalem, Israel.

\section{REFERENCES}

1. Papayannopoulou T, Kalmantis TH, Stamatayannopoulos G. (1979) Cellular regulation of hemoglobin switching: Evidence for inverse relationship between fetal hemoglobin synthesis and degree of maturity of human erythroid cells. Proc. Natl. Acad. Sci. U.S.A. 76: 6420-6424.

2. Alter BP, Weinberg RS, Goldberg JD, et al. (1983) Evidence for a clonal model for hemoglobin switching. Prog. Clin. Biol. Res. 134: 431-442.
3. Dalyot N, Fibach E, Rachmilewitz EA, Oppenheim A. (1992) Adult and neonatal patterns of human globin gene expression are recapitulated in liquid cultures. Exp. Hematol. 20: 1141-1145.

4. Fibach E, Manor D, Oppenheim A, Rachmilewitz EA. (1989) Proliferation and maturation of human erythroid progenitors in liquid culture. Blood 73: 100-103.

5. Dalyot N, Fibach E, Ronchi A, Rachmilewitz EA, Ottolenghi S, Oppenheim A. (1993) Erythropoietin triggers a burst of GATA-1 in normal human erythroid cells differentiating in tissue culture. Nucleic Acids Res. 21: 40314037.

6. Pevny L, Simon MC, Robertson E, et al. (1991) Erythroid differentiation in chimeric mice blocked by a targeted mutation in the gene for transcription factor GATA-1. Nature 349: 257-260.

7. Simon MC, Pevny L, Wiles MV, Keller G, Costantini F, Orkin SH. (1992) Rescue of erythroid development in gene targeted GATA-1 mouse embryonic stem cells. Nature Genet. 1: 92-98.

8. Orkin SH. (1990) Globin gene regulation and switching: Circa 1990. Cell 63: 665-672.

9. Philipsen S, Talbot D, Fraser P, Grosveld F. (1990) The beta-globin dominant control region: Hypersensitive site 2. E.M.B.O. J. 9: 2159-2167.

10. Fischer KD, Haese A, Nowock J. (1993) Cooperation of GATA-1 and SP1 can result in synergistic transcriptional activation or interference. J. Biol. Chem. 268: 23915-23923.

11. Minie ME, Kimura T, Felsenfeld G. (1992) The developmental switch in embryonic rho-globin expression is correlated with erythroid lineage-specific differences in transcription factor levels. Development 115: 1149-1164.

12. Orkin SH, Harosi FI, Leder P. (1975) Differentiation of erythroleukemic cells and their somatic hybrids. Proc. Natl. Acad. Sci. U.S.A. 72: 98-102.

13. Schreiber E, Matthias P, Muller MM, Schaffner W. (1989) Rapid detection of octamer binding proteins with 'mini-extracts' prepared from a small number of cells. $\mathrm{Nu}$ cleic Acids Res. 15: 6419-6436.

14. Chomczynski P, Sacchi N. (1987) Single-step method of RNA isolation by acid guanidinium thiocyanate-phenol-chloroform extraction. Anal. Biochem. 162: 156-159.

15. Leonard M, Brice M, Engel JD, Papayanno- 
poulou T. (1993) Dynamics of GATA transcription factor expression during erythroid differentiation. Blood 82: 1071-1079.

16. Mantovani R, Malgaretti N, Nicolis S, Ronchi A, Giglioni B, Ottolenghi S. (1988) The effects of HPFH mutations in the human $\gamma$-globin promoter on binding of ubiquitous and erythroid specific nuclear factors. Nucleic Acids Res. 16: 7783-7797.

17. Ronchi A, Nicolis S, Santoro C, Ottolenghi S. (1989) Increased Spl binding mediates erythroid-specific overexpression of a mutated (HPFH) $\gamma$-globin promoter. Nucleic Acids Res. 17: 10231-10240.

18. Alter BP, Coupal E, Forget BG. (1981) Globin chain electrophoresis for prenatal diagnosis of $\beta$-thalassemia. Hemoglobin 5: 357370.

19. Tsai FY, Keller G, Kuo FC, et al. (1994) An early haematopoietic defect in mice lacking the transcription factor GATA-2. Nature 371: 221-226.

20. Oppenheim A, Yaari A, Rund D, et al. (1990) Intrinsic potential for high fetal hemoglobin production in a Druz family with betathalassemia is due to an unlinked genetic determinant. Hum. Genet. 86: 175-180.

21. Oppenheim A, Katzir Y, Fibach E, Goldfarb A, Rachmilewitz EA. (1985) Hypomethylation of DNA derived from purified human erythroid cells correlates with gene activity of the beta-globin cluster. Blood 66: 12021207.

22. Thein SL, Sampietro $M$, Rohde $K$, et al. (1994) Detection of a major gene for heterocellular hereditary persistence of fetal hemoglobin after accounting for genetic modifiers. Am. J. Hum. Genet. 54: 214-228.

Contributed by S. H. Orkin on January 3, 1995.
23. Platt OS, Orkin SH, Dover G, Beardsly GP, Miller B, Nathan DG. (1984) Hydroxyurea enhances fetal hemoglobin production in sickle cell anemia. J. Clin. Invest. 74: 652656.

24. Perrine SP, Gindr GD, Faller DV, et al. (1993) A short-term trial of butyrate to stimulate fetal-globin-gene expression in the $\beta$-globin disorders. N. Engl. J. Med. 328: 81-86.

25. Fibach E, Burke LP, Schechter LN, Noguchi CT, Rodgers GP. (1993) Hydroxyurea increases fetal hemoglobin in cultured erythroid cells derived from normal individuals and patients with sickle cell anemia or betathalassemia. Blood 81: 1630-1635.

26. Fibach E, Prasanna P, Rodgers GP, Samid D. (1993) Enhanced fetal hemoglobin production by phenylacetate and 4-phenylbutyrate in erythroid precursors derived from normal donors and patients with sickle cell anemia and beta-thalassemia. Blood 82: 2203-2209.

27. Grosveld F, Blom van Assendelft G, Greaves DR, Kollias G. (1987) Position-independent, high-level expression of the human betaglobin gene in transgenic mice. Cell 51: 975985.

28. Forrester WC, Epner E, Driscoll MC, et al. (1990) A deletion of the human beta-globin locus activation region causes a major alteration in chromatin structure and replication across the entire beta-globin locus. Gene. Dev. 4: 1637-1649.

29. Cao SX, Gutman PD, Dave HP, Schechter AN. (1989) Identification of a transcriptional silencer in the $5^{\prime}$-flanking region of the human epsilon-globin gene. Proc. Natl. Acad. Sci. U.S.A. 86: 5306-5309. 